\title{
Infective aortic valve endocarditis with root abscess formation: a mitral sparing root-Commando operation
}

Quintana, Eduard ; Mestres, Carlos A ; Sandoval, E ; Ibáñez, C ; Van Hemelrijck, M ; Pomar, Jose L

DOI: https://doi.org/10.21037/acs.2019.06.09

Posted at the Zurich Open Repository and Archive, University of Zurich ZORA URL: https://doi.org/10.5167/uzh-186879

Journal Article

Published Version

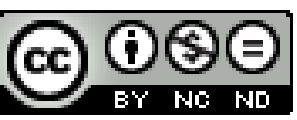

The following work is licensed under a Creative Commons: Attribution-NonCommercial-NoDerivatives 4.0 International (CC BY-NC-ND 4.0) License.

Originally published at:

Quintana, Eduard; Mestres, Carlos A; Sandoval, E; Ibáñez, C; Van Hemelrijck, M; Pomar, Jose L (2019). Infective aortic valve endocarditis with root abscess formation: a mitral sparing root-Commando operation. Annals of Cardiothoracic Surgery, 8(6):711-712.

DOI: https://doi.org/10.21037/acs.2019.06.09 


\title{
Infective aortic valve endocarditis with root abscess formation: a mitral sparing root-Commando operation
}

\author{
Eduard Quintana ${ }^{1}$, Carlos A. Mestres ${ }^{2}$, Elena Sandoval ${ }^{1}$, Cristina Ibáñez ${ }^{3}$, Mathias Van Hemelrijck ${ }^{2}$, \\ José Luis Pomar ${ }^{1}$ \\ ${ }^{1}$ Department of Cardiovascular Surgery, Cardiovascular Institute, Hospital Clínic de Barcelona, University of Barcelona, Institut d'Investigacions \\ Biomèdiques August Pi i Sunyer (IDIBAPS), Barcelona, Spain; ${ }^{2}$ Department of Cardiovascular Surgery, University Hospital Zürich, Zürich, \\ Switzerland; ${ }^{3}$ Department of Anesthesia, Hospital Clínic de Barcelona, Barcelona, Spain \\ Correspondence to: Eduard Quintana, MD, PhD, FECTS. Department of Cardiovascular Surgery, Cardiovascular Institute, Hospital Clínic de \\ Barcelona, University of Barcelona, C/Villarroel 170 CP, 08036, Barcelona, Spain. Email: equintan@clinic.cat.
}

Submitted May 11, 2019. Accepted for publication Jun 18, 2019.

doi: 10.21037/acs.2019.06.09

View this article at: http://dx.doi.org/10.21037/acs.2019.06.09

\section{Clinical vignette}

A 59-year-old male with rheumatoid arthritis treated with methotrexate and Staphylococcus epidermidis late prosthetic valve endocarditis is referred with new atrioventricular block. Echocardiography confirms aortic root abscess with intervalvular fibrosa (IVF) involvement and severe aortic prosthesis stenosis (gradient $65 / 46 \mathrm{mmHg}$ ) but no major perivalvular leak. External computed tomography (CT) scan demonstrated recent visceral and renal embolism. This patient had an EuroSCORE II of $28 \%$. Surgery was performed within the first 12 hours after admission to our center. A cryopreserved aortic homograft was obtained from our local cardiovascular tissue bank (1). The homograft was transported and stored in a dry-shipper with nitrogen in gas phase $\left(-140^{\circ} \mathrm{C}\right)$ and readily available as dictated by operative intracardiac findings.

\section{Surgical techniques}

\section{Preparation}

A pulmonary artery catheter is used in view of complex intracardiac reconstruction requiring a potentially prolonged aortic cross clamp. Central cannulation remains the first choice in the absence of increased chest re-entry risk.

\section{Exposition}

The decision to proceed with double venous cannulation is based on the eventual access to the right atrium to explore further endocarditic involvement or improve exposure and facilitate reconstruction beyond the aortic root involvement. Timing and location of left sided vent catheter is decided according to the presence of vegetations to prevent intraoperative embolism. Myocardial protection is achieved with blood cardioplegia.

\section{Operation}

After excision of the infected prosthesis, annular debridement is performed. Any suspicious tissue will be resected and the necessary reconstruction judged afterwards (video). When annular anatomy precludes securement of a sutured valve prosthesis then an aortic root homograft is our first choice. Extensive coronary mobilization ensures complete subcoronary debridement and improved homograft securement. When the IVF is extensively involved in conjunction with the anterior mitral leaflet this operation is best performed through the roof of the left atrium. The reconstruction of the roof of the left atrium, IVF and mitral leaflet is achieved by a tailored single bovine pericardial patch. In the case described herein, a posterior mitral annuloplasty is added to the reconstruction to increase the coaptation height but avoiding iatrogenic systolic anterior motion. Furthermore, this maneuver can decrease the risk of late onset mitral regurgitation in the event the pericardial patch biology alters the surface of the anterior leaflet with time. Trigonal securement is key to avoid bleeding and mitral detachment. An aortic root homograft is aligned trigone to trigone with the 
reconstructed mitral structures. The suture line is placed at a higher plane from the attachment of the pericardium to the mitral leaflet at the newly created atrial roof (on top of pericardial patch). The remaining root securement is obtained also with $3 / 0$ polypropylene semi continuous in the debrided left ventricular outflow tract. The coronary ostia are reimplanted and the operation is completed.

\section{Completion}

Blood pressure during the first 6-12 postoperative hours is maintained with a target systolic blood pressure around $100 \mathrm{mmHg}$. Only low dose norepinephrine support was required and early extubation was achieved. The patient had a favorable recovery, completing 4 weeks of intravenous antibiotics with a predischarge echocardiogram demonstrating anatomic reconstruction with normal bivalve function. He is currently leading an unrestricted life.

\section{Comments}

\section{Clinical results and advantages}

Aortic root abscess is known to be associated with increased mortality in patients undergoing surgery for active left-sided endocarditis (2). Patients with involvement of the IVF with extension towards the mitral valve constitute a further higherrisk population that will most likely face dismal prognosis without surgery. Many variants of the reconstruction of the aortic root and IVF (also known as the Commando operation) facilitate reproducible, complex repairs to grant survival and avoid the need for urgent cardiac transplantation. Structural involvement of the aortic root and mitral valve as well as the preoperative patient condition leads to the need to use a tailored approach selecting from many available options. In brief, when the aortic root is spared beyond the IVF region, a double valve replacement with IVF reconstruction may be enough (3). However, if root involvement dictates the need for root replacement, then a root-Commando with mitral replacement (4) or sparing of the mitral valve (5) may be the only reconstructive options. With an aggressive debridement philosophy, we have not experienced relapses of the original infection in the last 5 years. During the same time frame, survival for Commando operation variants has been above 94\% at Hospital Clínic de Barcelona in patients that would otherwise face a foreseeable demise. The need for a timely referral to a specialized endocarditis team is mandatory.

\section{Caveats}

It is important to note that the mitral-sparing Commando operation has been barely reported in the surgical literature and the need to judge its appropriateness at long term followup remains. However, at this point, it seems arguable that a technically impeccable operation should result in a durable valve repair. Whichever approach is chosen, it is key to obtain full debridement before implanting any valve substitute.

\section{Acknowledgments}

To the Cardiovascular Surgery Department Personnel, the Hospital Clínic Endocarditis Team and to the Central Catalonia 10 Endocarditis Team investigators.

\section{Footnote}

Conflicts of Interest: The authors have no conflicts of interest to declare.

\section{References}

1. Mestres CA, Agusti E, Martinez A, et al. Cardiovascular tissue banking in the non-cadaveric setting: ten-year experience of a university hospital-based bank with active organ donation program. J Heart Valve Dis 2000;9:523-9.

2. Leontyev S, Davierwala PM, Krögh G, et al. Early and late outcomes of complex aortic root surgery in patients with aortic root abscesses. Eur J Cardiothorac Surg 2016;49:447-54; discussion 454-5.

3. David TE, Kuo J, Armstrong S. Aortic and mitral valve replacement with reconstruction of the intervalvular fibrous body. J Thorac Cardiovasc Surg 1997;114:766-71; discussion 771-2.

4. Pettersson GB, Hussain ST, Ramankutty RM, et al. Reconstruction of fibrous skeleton: technique, pitfalls and results. Multimed Man Cardiothorac Surg 2014. doi: 10.1093/mmcts/mmu004.

5. Tomšic A, Schneider AW, Palmen M, et al. Extensive infective endocarditis of the aortic root and the aorticmitral continuity: a mitral valve sparing approach $\dagger$. Eur J Cardiothorac Surg 2017;51:1100-7.

Cite this article as: Quintana E, Mestres CA, Sandoval E, Ibáñez C, Van Hemelrijck M, Pomar JL. Infective aortic valve endocarditis with root abscess formation: a mitral sparing rootCommando operation. Ann Cardiothorac Surg 2019;8(6):711712. doi: $10.21037 /$ acs.2019.06.09 